\title{
Play(code): Uma proposta para estimular o aprendizado de Lógica de Programação
}

\author{
Glívia A. R. Barbosa ${ }^{1}$, Ismael S. Silva ${ }^{1}$, Flávio R. S. Coutinho ${ }^{1}$, Pedro Froes ${ }^{1}$, \\ Rafael Barbosa $^{1}$, Felipe Freitas ${ }^{1}$, Vitória M. P. do Nascimento ${ }^{1}$, Mariana \\ Bulgarelli', Gustavo Marques ${ }^{1}$, João P. Renan², Paula M. Ribeiro², Joice Sena, \\ Daniel Franco ${ }^{1}$ \\ ${ }^{1}$ Departamento de Computação - Centro Federal de Educação Tecnológica de Minas \\ Gerais (CFET-MG) \\ Av. Amazonas, 7675 - Nova Gameleira - Belo Horizonte, MG - Brasil \\ ${ }^{2}$ Departamento de Ciência da Computação - Universidade Federal de Minas Gerais \\ (UFMG) \\ Av. Antônio Carlos, 6627 - Prédio do ICEx Pampulha, Belo Horizonte, MG - Brasil

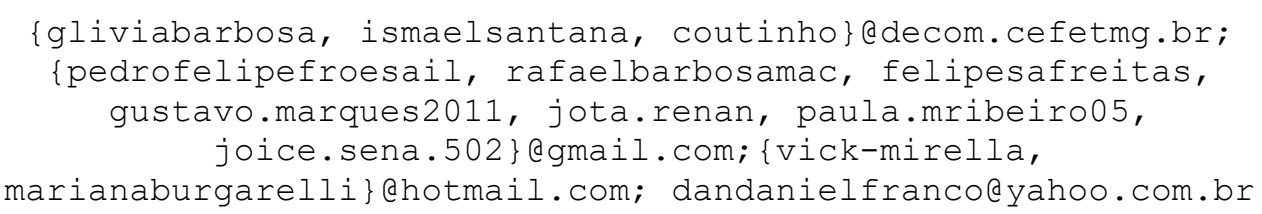

Resumo. Este artigo descreve o projeto e os resultados parciais de uma pesquisa em andamento que busca estimular o aprendizado de Lógica de Programação, por meio de uma plataforma online gamificada e colaborativa.

\section{Introdução}

O uso da tecnologia como suporte ao ensino tem alterado os padrões pedagógicos e de aprendizagem. Com o advento dos serviços web e a adoção em massa dos aplicativos (apps) móveis, as pessoas encontram na tecnologia diferentes possibilidades de acesso à informação e obtenção do conhecimento que transcendem os limites da sala de aula [Clark e Mayer, 2012].

Uma área de conhecimento que tem utilizado a tecnologia como complemento ao ensino é a Lógica de Programação. Segundo Clark e Mayer (2012), programar é uma tarefa desafiadora e complexa, logo, foi necessário fazer uso da própria tecnologia para repensar os modelos tradicionais de educação e descobrir novas formas de ensino que facilitem o aprendizado da Lógica de Programação.

Visando contornar esse desafio, é possível encontrar serviços web e apps móveis que buscam auxiliar no aprendizado de Lógica de Programação (e.g., Code Academy ${ }^{1}$ e Encode $^{2}$ ). Essas tecnologias podem ser utilizadas por professores, como um recurso complementar ao ensino desse conteúdo, bem como um recurso para que os usuários interessados possam iniciar os estudos em Lógica de Programação [Clark e Mayer, 2012].

Entretanto, apesar da existência dessas tecnologias, existe o desafio e a demanda de propor plataformas/apps que, além de expor o conteúdo, estimulam e motivam os

\footnotetext{
${ }^{1}$ Code Academy - https://www.codecademy.com/

${ }^{2}$ Encode - https://play.google.com/store/apps/details?id=com.upskew.encode\&hl=en
} 
VII Congresso Brasileiro de Informática na Educação (CBIE 2018)

Anais do XXIX Simpósio Brasileiro de Informática na Educação (SBIE 2018)

usuários na prática e no aprendizado contínuo da Lógica de Programação. Nesse sentido, profissionais e pesquisadores da Computação têm investigado a aplicabilidade da adoção de estratégias de qualidade de uso (e.g., sociabilidade, gamificação e colaboração) nos modelos de interface e interação das tecnologias voltadas para o ensino com o intuito de incentivar a aceitação e o uso contínuo dessas plataformas/apps [da Silva et al., 2015].

Motivados por esse cenário, este trabalho apresenta a proposta e os resultados parciais de uma pesquisa que busca estimular o aprendizado de Lógica de Programação, por meio de uma plataforma online gamificada (i.e., que faz uso de gamificação) e colaborativa, denominada Play(code). O objetivo do Play(code) é oferecer um ambiente de ensino que fomenta o aprendizado, autodidata ou guiado por um tutor, de Lógica de Programação, por meio de um computador pessoal ou dispositivo móvel.

Em termos de contribuições, essa pesquisa é relevante uma vez que disponibiliza uma plataforma para auxiliar no ensino de Lógica de Programação, por meio de recursos que estimulam o aprendizado de um conteúdo considerado desafiador e complexo. Além disso, o trabalho discute a aplicabilidade de adotar qualidades de uso como, sociabilidade, gamificação e colaboração, nos sistemas educacionais como estratégias para estimular e motivar o uso continuo desse tipo de tecnologia.

\section{Metodologia}

Para alcançar o objetivo proposto, a seguinte questão de pesquisa está sendo investigada: "Como estimular o aprendizado de Lógica Programação por meio de uma plataforma online?". A metodologia adotada para responder essa questão consiste em uma abordagem dividida em cinco etapas. Sendo que dessas, três já foram concluídas. Inicialmente, foi realizado um levantamento e uma caracterização dos sistemas educacionais existentes focados no ensino de Lógica de Programação. Por meio dessa caraterização foi possível identificar as vantagens e os limites desses sistemas.

Em seguida, foram levantadas, na literatura, as estratégias de qualidade de uso que visam estimular e motivar a utilização contínua de sistemas interativos. Uma vez identificadas, essas estratégias foram avaliadas quanto a aplicabilidade das mesmas para o contexto de sistemas educacionais. Essa análise permitiu identificar as estratégias de colaboração, gamificação e user experience (UX) a serem adotadas no modelo de interface e interação do Play(code). Posteriormente, os requisitos funcionais, não funcionais e a arquitetura do Play(code) foram definidos e especificados, visando alcançar objetivo da plataforma.

A quarta fase, em andamento, consiste no desenvolvimento do Play(code). A plataforma está sendo desenvolvida em módulos e cada nova versão é verificada por testadores e avaliadas por especialistas em Interação Humano Computador (IHC). Finalizado o desenvolvimento, o Play(code) será avaliado junto aos usuários, tanto em relação a suas funcionalidades, quanto em relação ao projeto de interface e interação. Essas avalições são importantes uma vez que o principal objetivo da plataforma é estimular a experiencia de uso e o aprendizado por meio da plataforma.

\section{A Plataforma Play(code)}

A plataforma Play(code) consiste em um ambiente de ensino que estimula e fomenta o aprendizado, autodidata ou guiado por um tutor, de Lógica de Programação. Essa plataforma poderá ser acessada por meio de um computador pessoal ou dispositivo móvel 
VII Congresso Brasileiro de Informática na Educação (CBIE 2018)

Anais do XXIX Simpósio Brasileiro de Informática na Educação (SBIE 2018)

e será composta por três módulos.

O primeiro, consiste no módulo de elaboração de questões e lições relacionadas ao conteúdo de Lógica de Programação. As questões vinculadas ao Play(code) serão criadas e gerenciadas de forma colaborativa. $\mathrm{O}$ segundo módulo contempla recursos para a resolução de questões e lições. Esse módulo será gamificado e colaborativo, visando um maior engajamento e interação contínua dos usuários na tarefa de solucionar questões relacionadas a Lógica de Programação.

Por sua vez, o terceiro módulo refere-se ao acompanhamento do desempenho, no qual o usuário poderá visualizar seu aproveitamento nas tarefas já realizadas e, além disso, compartilhar e comparar seus resultados com outros usuários. A Figura 1, ilustra as telas de login, listagem de módulos e lições, bem como a interface que o usuário interage com uma questão por meio do Play(code).

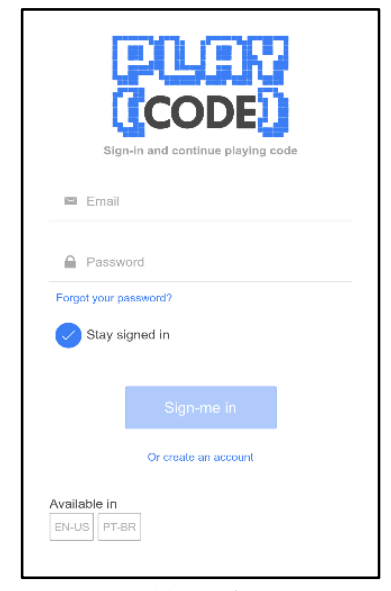

(a) Login

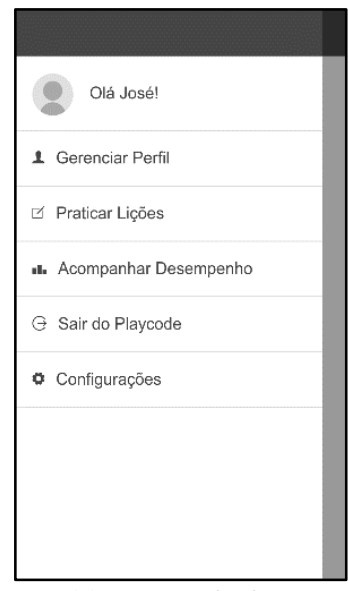

(b) Menu Principal

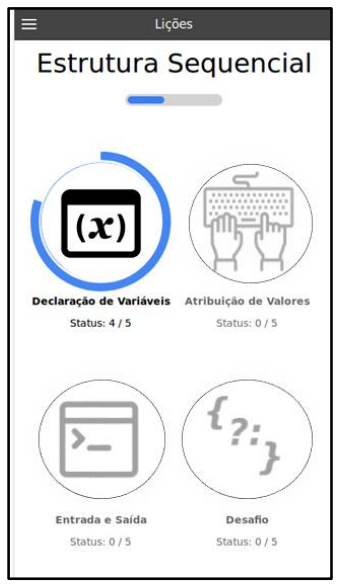

(c) Listagem de Lições do

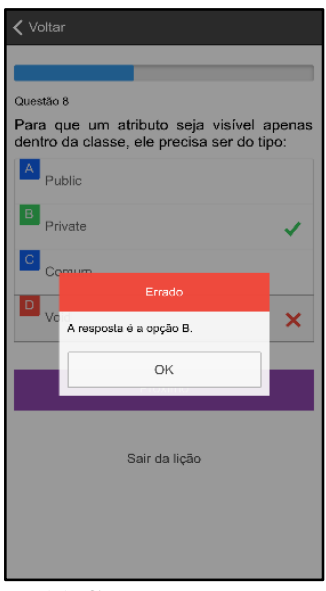

(d) Correção de uma questão

Figura 1. Exemplos de interface do Play(code)

\section{Considerações Finais}

O projeto Play(code) impacta nas áreas de Computação e Educação. No âmbito da Educação, a plataforma proposta poderá auxiliar os alunos no aprendizado de Lógica de Programação, além de estimular diversas pessoas a buscarem conhecimento nessa área. De forma análoga, os professores poderão utilizar a plataforma como complemento em sua metodologia de ensino. Sendo assim, o Play(code) contribuirá tanto para o modelo de educação à distância, quanto para o presencial, o que, segundo Moran (2002), é uma característica relevante de sistemas de apoio ao ensino.

Para a Computação, esse projeto impacta no avanço das pesquisas que visam consolidar e definir as limitações do e-learning como modelo de ensino de Lógica de Programação. De forma complementar, o projeto contribui com insights sobre a aplicabilidade das estratégias de colaboração, gamificação e UX para estimular e motivar o uso contínuo de sistemas educacionais. Essa contribuição é relevante porque pesquisadores da área de IHC reforçam a necessidade discutir a aplicabilidade dessas estratégias para delinear suas vantagens e desvantagens em diferentes domínios de sistemas, inclusive os educacionais [da Silva, et al., 2015].

Como direções futuras, os próximos passos dessa pesquisa consistem em (1) 
VII Congresso Brasileiro de Informática na Educação (CBIE 2018)

Anais do XXIX Simpósio Brasileiro de Informática na Educação (SBIE 2018)

finalizar o desenvolvimento da plataforma Play(code) e (2) avaliar e discutir o impacto dessa plataforma como um recurso para auxiliar e estimular o aprendizado de Lógica de Programação.

\section{Referências}

Clark, R. C. and Mayer, R. E. (2012). E-Learning and the Science of Instruction: Proven Guidelines for Consumers and Designers of Multimedia Learning (3nd ed.). Pfeiffer \& Company.

da Silva, J. C., Oliveira, E. R., Barbosa, G. A. R. (2015). Caracterização de Estratégias de Gamificação em Aplicativos Móveis Educacionais: Um Estudo de Caso do Aplicativo Duolingo. In XIV IHC, 2015.

Moran, J. 2002. O que é educação a distância - Novos caminhos do ensino a distância, CEAD - Centro de Educação a Distância. SENAI, Rio de Janeiro, ano 1, n.5, pag 1-3. 